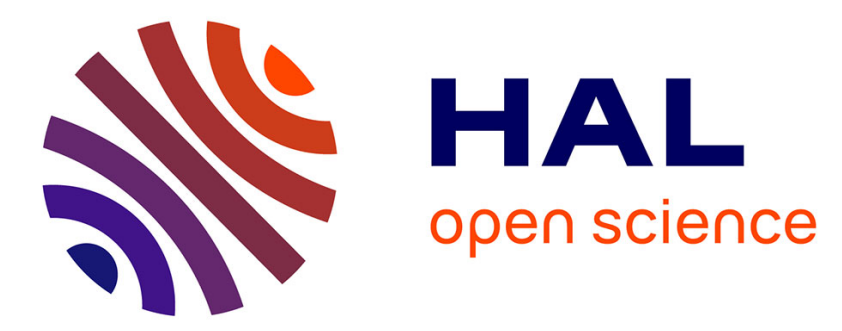

\title{
Condensate fragmentation as a sensitive measure of the quantum many-body behavior of bosons with long-range interactions
}

Uwe R Fischer, Axel Lode, Budhaditya Chatterjee

\section{- To cite this version:}

Uwe R Fischer, Axel Lode, Budhaditya Chatterjee. Condensate fragmentation as a sensitive measure of the quantum many-body behavior of bosons with long-range interactions. Physical Review A, 2015, 91, pp.063621. 10.1103/PhysRevA.91.063621 . hal-02369898

\section{HAL Id: hal-02369898 https://hal.science/hal-02369898}

Submitted on 19 Nov 2019

HAL is a multi-disciplinary open access archive for the deposit and dissemination of scientific research documents, whether they are published or not. The documents may come from teaching and research institutions in France or abroad, or from public or private research centers.
L'archive ouverte pluridisciplinaire HAL, est destinée au dépôt et à la diffusion de documents scientifiques de niveau recherche, publiés ou non, émanant des établissements d'enseignement et de recherche français ou étrangers, des laboratoires publics ou privés. 


\title{
Condensate fragmentation as a sensitive measure of the quantum many-body behavior of bosons with long-range interactions
}

\author{
Uwe R. Fischer, ${ }^{1}$ Axel U. J. Lode, ${ }^{2}$ and Budhaditya Chatterjee ${ }^{1}$ \\ ${ }^{1}$ Department of Physics and Astronomy, Center for Theoretical Physics, Seoul National University, 151-747 Seoul, Korea \\ ${ }^{2}$ Condensed Matter Theory and Quantum Computing Group, Department of Physics, University of Basel, Klingelbergstrasse 82, \\ 4056 Basel, Switzerland
}

(Received 24 February 2015; published 18 June 2015)

\begin{abstract}
The occupation of more than one single-particle state, and hence the emergence of fragmentation, is a many-body phenomenon occurring for systems of spatially confined strongly interacting bosons. In the present study, we investigate the effect of the range of the interparticle interactions on the fragmentation degree of one- and two-dimensional systems in single wells. We solve the full many-body Schrödinger equation of the system using the recursive implementation of the multiconfigurational time-dependent Hartree for bosons method (R-MCTDHB). The dependence of the degree of fragmentation on dimensionality, particle number, areal or line density, and interaction strength is assessed. For contact interactions, it is found that the fragmentation is essentially density independent in two dimensions. However, fragmentation increasingly depends on density the more long ranged the interactions become. At fixed particle number $N$, the degree of fragmentation is increasing when the density is decreasing, as expected in one spatial dimension. We demonstrate that this, nontrivially, remains true also for long-range interactions in two spatial dimensions. We, finally, find that fragmentation in a single well is a mesoscopic phenomenon: Within our fully self-consistent approach, the degree of fragmentation, to a good approximation, decreases universally as $N^{-1 / 2}$ when only $N$ is varied.
\end{abstract}

DOI: 10.1103/PhysRevA.91.063621

PACS number(s): 03.75.Gg, 03.75.Nt

\section{INTRODUCTION}

The many-body behavior of systems of interacting bosons can, in many cases, be essentially characterized by a single "diluteness parameter" expressing the importance of interactions [1]. For most experimentally produced samples of ultracold bosonic gases, describing them by such a (small) diluteness parameter essentially yields the correct physics because the mean interparticle distance is much smaller than the range of the interparticle interaction potential. Archetypical theoretical methods employing the diluteness of the gas are the Gross-Pitaevskiir mean-field theory [1] and, with (quantum) fluctuations incorporated to lowest order, Bogoliubov theory [2]. These approaches assume that a single macroscopically occupied orbital forms the many-body state and hence that a single creation operator in the field-operator expansion is sufficient.

In particular, in one and in three spatial dimensions (1D and 3D), diluteness criteria are well established for integrable as well as nonintegrable interparticle interactions with a potential $V_{\text {int }}(r) \propto 1 / r^{n}$ [3]. Integrability here means the (infrared) convergence of the integral $\int V_{\mathrm{int}}(r) d^{D} r$, implying $n>D$, where $D$ is the spatial dimension. In the following, we use the terminology of interactions being short and long range interchangeably with them being integrable and nonintegrable, respectively. It is a widely accepted fact that for short-range interactions, the gas is more dilute, and theories such as Bogoliubov mean field are applicable for low densities in 3D and, conversely, for large densities in 1D. For long-range interactions, in particular for Coulomb interactions, the gas cannot be considered dilute when the density decreases in 3D. A consequence of this fact is, for example, the formation of a Wigner crystal [4] at very low densities. For sufficiently high densities, the interacting gas can, conversely, be described by Bogoliubov theory [5]. On the other hand, in one dimension, high densities, in combination with Coulomb interactions $(n=1)$, lead to a strongly correlated Tonks-Girardeau gas [6]. For very large repulsive contact interactions in $1 \mathrm{D}$ the density profile can be captured by either a Thomas-Fermi or a TonksGirardeau local equation of state for high and low densities, respectively [7].

Two spatial dimensions (2D), which are the main focus in what follows, represent a crossover case between the two limiting behaviors of $1 \mathrm{D}$ and $3 \mathrm{D}$ with respect to the effective diluteness of the gas and therefore constitute a sensitive probe for many-body effects. In 2D, correlations can potentially play a significant role for both integrable and nonintegrable interactions. For integrable interactions such as contact pseudopotentials it is known that the diluteness parameter in $2 \mathrm{D}$ is essentially independent of the areal density $\rho$, involving a double logarithm $\ln \ln \left[\rho a_{\text {eff }}^{2}\right]$, where $a_{\text {eff }}$ is the effective range of the two-body interaction [8]. In the infinitely extended homogeneous gas, two dimensions represent the marginal case for the existence of condensates [9-12]; at any finite temperature condensation is absent, while at zero temperature condensation is possible, albeit for extremely small densities [13]. Two-dimensional Bose gases have been comprehensively studied for short-range interactions (see the review in [14]). However, comparatively little is known for long-range interactions, apart from the case where gauge fields of constant large flux are externally applied to twodimensional electron gases, where the theoretical foundation of the fractional quantum Hall effect in the form of the eponymous Laughlin wave function [15] has spurred both tremendous experimental and theoretical activity [16]. We also note in this regard that the many-body physics of dipolar bosons in 2D (see, for instance, [17]) does not qualify with the presently employed terminology as being genuinely "long range" due to the integrability of the interaction potential of (perpendicularly polarized) dipoles in two spatial dimensions, 
implying in particular the existence of a well-defined Fourier transform of $V_{\text {int }}(r)$ at $k=0$ [18].

The question how the diluteness of a bosonic many-body system depends on the power-law tail of the interaction potential has thus not yet been addressed generally, in particular for the two-dimensional case. In the present work, we aim to fill this gap by employing the recursive implementation of the multiconfigurational time-dependent Hartree for bosons method (R-MCTDHB) [19] to solve the many-body problem with both integrable and nonintegrable interaction potentials of varying power law $n$.

The phenomenon of fragmentation (see, e.g., [20-24]), in which more than one field-operator mode becomes macroscopically occupied, so that Bogoliubov mean-field theory necessarily breaks down, has previously in particular been extensively studied in one dimension. For instance, a stepwise increase of the number of fragments takes place in an asymmetric double well [25]. Because of the strongly increased demand in computational resources the two-dimensional and three-dimensional cases have only recently attracted attention due to the availability of novel numerical methods (see, for instance, [19]). Fragmentation has been found in the case of an effective potential barrier [26] induced by the long-range interactions or the dynamics above a condensed ground state [27].

We focus below on studying fragmentation as an "intrinsic" property of the many-body system in a single (e.g., hard-wall or harmonic) trap. In this case, fragmentation is termed intrinsic because it is not assisted by the one-body potential in the Hamiltonian. This is in contrast to the cases where fragmentation is "extrinsic," i.e., assisted by the one-body potential as, for instance, in double wells [28,29] or in its periodic extension in optical lattices [30,31]. We refer to intrinsic fragmentation as a genuine many-body effect because it originates solely in the interparticle interactions and thus does not essentially depend on the way in which the bosons are confined. It is also important to note in this regard that the actual detection of fragmentation in a single trap requires significantly more experimental effort than in double wells or optical lattices. Fragmentation in the superfluid-Mott transition on optical lattices is detected by the decrease in the visibility of the structure factor peaks [31]. However, this approach employs a measure of first-order coherence that is not conclusive for the determination of the eigenvalues of the single-particle density matrix [32] and hence does not yield the desired information on fragmentation [33]. This stems essentially from the fact that the macroscopically occupied natural orbitals will significantly overlap in a potentially complicated fashion, which is distinct from the multiple-well scenario deep in the Mott regime, where they are well separated. Unequivocally assigning fragmentation by just measuring first-order correlations will therefore, in general, not be possible in single traps, particularly when the degree of fragmentation is comparatively small.

Exactly solvable cases for benchmarking the numerics are very scarce and significantly more specialized in spatial dimensions higher than 1. For the one-dimensional case, exactly solvable systems include the Tonks-Girardeau gas and the Lieb-Liniger and Calogero-Sutherland models, as reviewed in [34]. Analytical ground-state solutions in two dimensions have been obtained, for instance, by constructing a variant of Laughlin's wave function for particles in an (effective) magnetic field with hard-core interaction [35] or for the variant of the Calogero model considered in [36]. In any spatial dimension, Richardson's pairing model employing a contact pairing interaction potential allows for exact solutions [37]. Numerically, it has been shown using the harmonic interaction model [38,39] and its time-dependent generalization that the approach of (R-)MCTDHB can solve the generally time-dependent many-body problem to an, in principle, arbitrary degree of accuracy in 1D [40] and also in 2D [41].

In what follows, we exploit the fact that condensate fragmentation is a genuine many-body phenomenon whose very existence relies on both first- and higher-order correlations. We show that when the gas is no longer dilute, and hence Bogoliubov theory breaks down, depends strongly on the power law of two-body interactions. We then argue that a system can be considered dilute if and only if it is accurately described by a mean-field theory because fragmentation is absent. On the other hand, a system is said to be not dilute (or equivalently strongly correlated) if fragmentation is significant, say, on the order of 5\%-10\%. We thus use the degree of fragmentation as an indicator of the "many-bodyness" of an interacting quantum gas: When fragmentation becomes significant, the description of the system on an effective single-particle level, that is, within mean-field theories becomes inapplicable.

We will show that, indeed, the degree of fragmentation is both small and essentially density independent for contact interactions. However, the more long ranged the interactions become, the more significantly the fragmentation degree depends on the density and exceeds the contact interaction value at sufficiently small densities. The degree of fragmentation thus increases when the density of particles in the gas decreases. While this is a well-known fact in one dimension and for contact interactions, in two dimensions and for longrange interactions this result is a nontrivial manifestation of many-body physics.

In addition, we make explicit that besides the density the total number of particles $N$ is an independent parameter with which to assess the mean-field behavior of the system. We demonstrate that for any power law of interactions, the degree of fragmentation, and thus the degree to which the genuine quantum many-body nature of the interacting system is manifest, is reduced with increasing $N$. This markedly differs from the functional behavior of the degree of fragmentation upon varying $\rho$, which strongly depends on spatial dimension and the power law of interactions.

\section{MODEL AND METHODS}

\section{A. Setup}

The $N$-body Hamiltonian reads

$$
\hat{H}=\sum_{i=1}^{N} \hat{h}\left(\boldsymbol{r}_{i}\right)+\sum_{i<j=1}^{N} \hat{V}_{\mathrm{int}}\left(\boldsymbol{r}_{i}, \boldsymbol{r}_{j}\right) .
$$

Here, $\hat{h}\left(\boldsymbol{r}_{i}\right)=-\frac{1}{2 m} \nabla_{i}^{2}+V_{\text {ext }}\left(\boldsymbol{r}_{i}\right)$ is the one-body Hamiltonian that contains the kinetic and potential energy of the particle $i$, with $m$ being the boson mass, and $\hat{V}_{\text {int }}\left(\boldsymbol{r}_{i}, \boldsymbol{r}_{j}\right)$ is the two-body interaction for a pair of particles at $\boldsymbol{r}_{i}$ and $\boldsymbol{r}_{j}$. For reasons 
of computational convenience, we rescale the Hamiltonian by $\frac{\hbar^{2}}{2 m l^{2}}$. The length unit $l$, which can, for example, be chosen to represent a typical interparticle separation, thus defines the units we employ; all quantities in what follows are dimensionless.

We consider a two-dimensional spherically symmetric box potential specifically given by

$$
V_{\text {ext }}(r)= \begin{cases}0 & r \leqslant a, \\ 1000 & r>a\end{cases}
$$

The areal density for $N$ particles is thus given by $\rho=\frac{N}{\pi a^{2}}$. Note that most recent advances in engineering atom traps allow for box traps to a very good approximation, enabling the experimental study of the ground state of homogeneous Bose gases in various spatial dimensions $[42,43]$.

In view of the convergence issues for a true $\delta$ potential in two dimensions $[44,45]$, the contact interaction is modeled by a normalized Gaussian as follows:

$$
V_{\text {int }}^{\delta}\left(R=\left|\boldsymbol{r}_{i}-\boldsymbol{r}_{j}\right|\right)=g \frac{\exp \left(-\frac{R^{2}}{2 \sigma^{2}}\right)}{2 \pi \sigma^{2}},
$$

with width $\sigma=0.25$ chosen such that it reproduces the physics of a contact interaction [44]. The long-range interaction potential of two particles at $\boldsymbol{r}_{i}$ and $\boldsymbol{r}_{j}$, respectively, is modeled by the regularized expression

$$
V_{\mathrm{int}}\left(R=\left|\boldsymbol{r}_{i}-\boldsymbol{r}_{j}\right|\right)=\frac{g}{R^{n}+\Delta^{n}},
$$

where $\Delta=0.07$ is the cutoff and $n$ is the power law of the long-range interaction potential.

Fragmentation is computed by calculating the natural orbital occupation numbers $N_{i}$, which are the eigenvalues of the one-body density matrix

$$
\rho^{(1)}\left(\boldsymbol{r}^{\prime} \mid \boldsymbol{r}\right)=\sum_{\alpha} N_{\alpha} \phi_{\alpha}^{(\mathrm{NO}), *}\left(\boldsymbol{r}^{\prime}\right) \phi_{\alpha}^{(\mathrm{NO})}(\boldsymbol{r}),
$$

with the eigenvectors $\phi_{\alpha}^{(\mathrm{NO})}(\boldsymbol{r})$ representing the natural orbitals. The degree of fragmentation (for two modes) is then defined as [24]

$$
\mathcal{F}=1-\frac{\left|N_{1}-N_{2}\right|}{N},
$$

where $N_{1,2}$ are the two largest natural orbital occupation numbers, defined by Eq. (5). We note that we have verified that in the parameter ranges under investigation below, the occupation of a possible third orbital remains negligibly small, so that performing a truncation after two modes in the field-operator expansion is justified.

\section{B. Numerical method}

We use the multiconfigurational time-dependent Hartree method for bosons (MCTDHB) [23] in its recent recursive implementation (R-MCTDHB) [19] for the numerically demanding task of computing the solution of the many-body Schrödinger equation to an, in principle, arbitrarily large degree of accuracy. The MCTDHB method uses the following ansatz for the wave function:

$$
|\Psi\rangle=\sum_{\vec{n}} C_{\vec{n}}(t)|\vec{n} ; t\rangle .
$$

Here, $|\Psi\rangle$ is expanded as a linear combination of timedependent, fully variationally determined and symmetrized many-body basis states,

$$
|\vec{n} ; t\rangle=\prod_{\alpha=1}^{M} \frac{\left(b_{\alpha}^{\dagger}(t)\right)^{n_{\alpha}}}{\sqrt{n_{\alpha} !}}|\mathrm{vac}\rangle,
$$

where vector notation was invoked for the occupations, $\vec{n}=\left(n_{1}, \ldots, n_{M}\right)^{T}$, which fulfill $N=\sum_{\alpha} n_{\alpha}$. The many-body basis states $|\vec{n} ; t\rangle$ are obtained by applying a symmetrization operator to Hartree products that are built from at most $M$ distinct orthonormal orbitals $\left\{\phi_{\alpha}(\boldsymbol{r} ; t) ; \alpha=1, \ldots, M\right\}$. With this ansatz one tackles the time-dependent many-body Schrödinger equation, using the time-dependent variational principle [46]. As the result of the variation of the action functional, two sets of equations of motion, one nonlinear integro-differential set for the time evolution of the $M$ single-particle basis states or orbitals $\left\{\phi_{\alpha}(\boldsymbol{r} ; t) ; \alpha=1, \ldots, M\right\}$ and one linear set for the time evolution of the $\left(\begin{array}{c}N+M-1 \\ N\end{array}\right)$ coefficients $\left\{C_{\vec{n}}(t)\right\}$, are obtained; see Ref. [23] for a detailed discussion. The two sets are coupled because the time evolution of the orbitals depends on the matrix elements $\rho_{\alpha \beta}(t), \rho_{\alpha \beta \gamma \delta}(t)$, which are functions of the coefficients, whereas the time evolution of the coefficients depends on the matrix elements of the one-body and two-body Hamiltonian, $h_{\alpha \beta}, W_{\alpha \beta \gamma \delta}$. The simultaneous and self-consistent solution of the system of MCTDHB equations of motion for both $\left\{C_{\vec{n}}(t)\right\}$ and $\left\{\phi_{\alpha}(\vec{r} ; t)\right\}$ is equivalent to solving the full (time-dependent) many-boson problem if convergence with respect to the number of variational parameters is achieved (see $[40,41]$ ). This is a consequence of the formal exactness of the ansatz in Eq. (7); in the case of $M \rightarrow \infty$, it covers the full $N$-boson Hilbert space. Note that in the case of $M=1$, Eq. (7) is a mean-field product state, and therefore, the set of MCTDHB equations boils down to the time-dependent Gross-Pitaevskii equation [23].

\section{INTEGRABLE AND NONINTEGRABLE INTERACTIONS}

\section{A. Two-dimensional disk geometry}

\section{Fragmentation for various power laws of interaction}

Especially for the presently considered mesoscopic systems, $N$ and $\rho$ have distinct influence on the many-body state of the system. In the first-quantized form, $N$ is a fundamental parameter of the many-body Hamiltonian, while $\rho$ is the ratio $\rho=N / A$; here, the area $A$ depends on the external potential. A change in $N$ affects both the external potential and the interaction term in the Hamiltonian, Eq. (1), while changing $A$ requires changing only the external potential and leaves the interaction term of the Hamiltonian unchanged. Since the $N$ and $A$ dependences of the system are different, the value of $\rho$ does not uniquely determine the many-body wave function, which also explicitly depends on the value of $N$.

Hence, to analyze the density dependence of fragmentation independent from the influence of $N$, we keep $N=100$ fixed and first vary the area $A$ of the disk by changing its radius $a$ in the $2 \mathrm{D}$ box potential, Eq. (2).

The density dependence of fragmentation is assessed using a contact interaction and interactions with power laws 


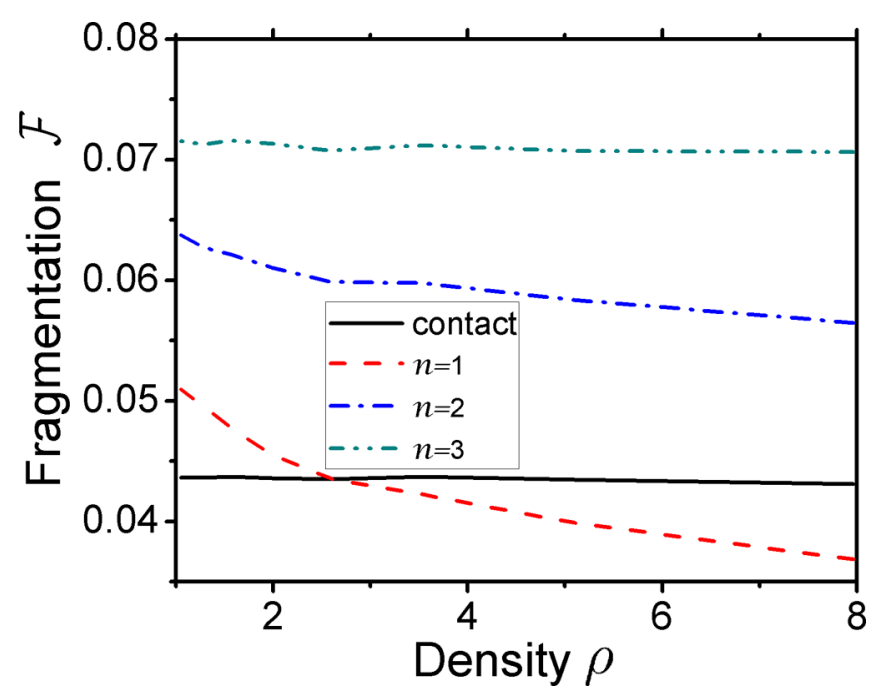

FIG. 1. (Color online) The variation of fragmentation with dimensionless $2 \mathrm{D}$ density $\rho=\frac{N}{\pi a^{2}}$ for $N=100$ particles, comparing contact interaction and interactions of power law $n=1,2,3$ at fixed $V_{\text {eff }}=2 \pi \times 11.6$.

$n=1,2,3$ in Eq. (4). In principle, a comparison between different interaction potentials can be made with respect to the same coupling constant $g$. However, a more useful comparison is obtained using the integrated measure of the 2D interaction potential $V_{\text {eff }} \equiv 2 \pi \int V_{\text {int }}(R) R d R(\sim g)$ since $V_{\text {int }}$ enters the many-body Hamiltonian as the kernel of the integrals $W_{\alpha \beta \gamma \delta}$ [see Ref. [23] and Eq. (9) below]. The coupling constants are then chosen such that they correspond to the same $V_{\text {eff }}$ for different interaction potentials. Physical realizations of $n=1$ in 1D and 2D are conceivable for Coulomb gases and for $n=3$ in gases of perpendicularly polarized dipoles [18]. For the Calogero-Sutherland model $(n=2)$ in $1 \mathrm{D}$, an ultracold gas implementation has been suggested in [47].

In Fig. 1, we compare the variation of fragmentation $\mathcal{F}$ with density $\rho$, holding $V_{\text {eff }}=2 \pi \times 11.6$ constant. For contact interaction (see Eq. (3)), there is hardly a clearly discernible variation of $\mathcal{F}$ with $\rho$. For long-range interactions $[n=1,2$ in Eq. (4)], on the other hand, the degree of fragmentation decreases with $\rho$. For the 2D integrable interactions with $n=3$ and $n>3$ (not shown), the influence of density on fragmentation is again minimal.

This leads us to conclude that the influence of density on the degree of fragmentation for two spatial dimensions depends on the integrability of the interaction potential; for integrable interaction (Eqs. (3) and (4) for $n \geqslant 3$ ), the variation of $\mathcal{F}$ with $\rho$ is minimal, while for nonintegrable interactions $[n=1,2$ in Eq. (4)], the fragmentation degree decreases with density. We also note that for $n=1$, the most long-ranged case, the extent of the decrease of $\mathcal{F}(\rho)$ is significantly stronger than for $n=2$; that is the more long ranged the interaction is, the stronger $\mathcal{F}$ varies with density. The $n=1$ curve, while starting from higher values for small densities, crosses the curve for contact interactions, decreasing to a lower value of $\mathcal{F}$ for higher densities. The $n=2$ curve again starts with a higher value of $\mathcal{F}$ for low density, decreasing slowly, and approaches the contact interaction curve for large densities. For $n=3$, $\mathcal{F}(\rho)$ is highest for all densities. Finally, for sufficiently large densities, the $n=1$ interaction is the least fragmented, and the integrable $n=3$ interaction is the most fragmented. We have verified that these qualitative features are similar for any number of particles $N$ and interaction coupling $g$ with only the value of fragmentation $\mathcal{F}$ being different.

\section{Effective Thomas-Fermi parameters}

The degree of fragmentation for a fixed $N$ depends on the interplay between the kinetic energy and interaction energy. Thus, an important measure in determining the fragmentation of the system is the Thomas-Fermi parameter represented by the interaction energy and kinetic energy, $P_{\mathrm{TF}}=\frac{E_{\mathrm{int}}}{E_{\mathrm{kin}}}$.

Figure 2 shows the variation of the Thomas-Fermi parameter $P_{\mathrm{TF}}$ with density $\rho$ for fixed $N=100$. One can observe a significant difference between integrable and nonintegrable interactions, particularly in the low-density regime. For contact interaction, there is a very slow decrease of $P_{\mathrm{TF}}$ with $\rho$ throughout the entire density regime; for the integrable $n=3$, $P_{\mathrm{TF}}$ remains roughly constant with $\rho$ as well. A strikingly different functional dependence is seen for $n=1$. While the large $\rho$ behavior is similar to that of contact interactions, for small $\rho$, there is a very sharp increase of $P_{\mathrm{TF}}$ with decreasing density. A similar tendency (albeit to a smaller degree) is observed for $n=2$. This strong increase of $P_{\mathrm{TF}}$ for decreasing density, implying the stronger influence of the interaction energy as compared to the kinetic energy, manifests itself in the increasing degree of fragmentation with lowering density seen for nonintegrable interactions (see Fig. 1).

To obtain a qualitative understanding of the abovediscussed dependence on $\rho$, we note that in the mean-field regime for contact interactions, both the interaction and kinetic energy go linearly with density and $P_{\mathrm{TF}}$ does not vary with density. For many-body systems, however, in general, the equally linear dependence of kinetic and interaction energy does not strictly hold, and deviations are observed. In our case, the interaction energy varies slightly faster with $\rho$ than

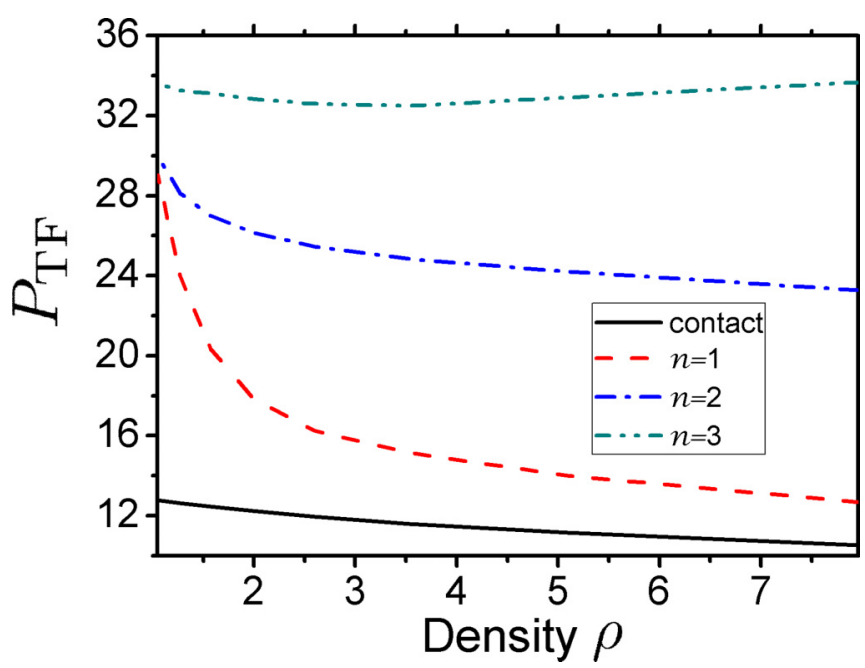

FIG. 2. (Color online) The variation of the Thomas-Fermi parameter $P_{\mathrm{TF}}(\rho)$ with dimensionless $2 \mathrm{D}$ density $\rho$ for a fixed $N=$ 100 for contact interaction and long-range interactions of degree $n=1,2,3$ in two spatial dimensions. 


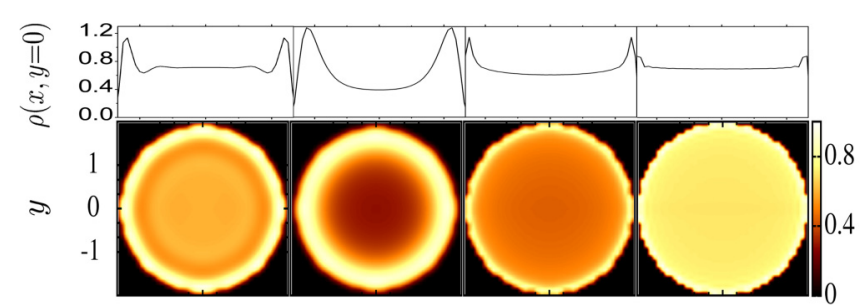

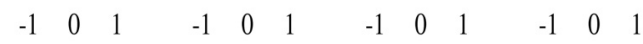

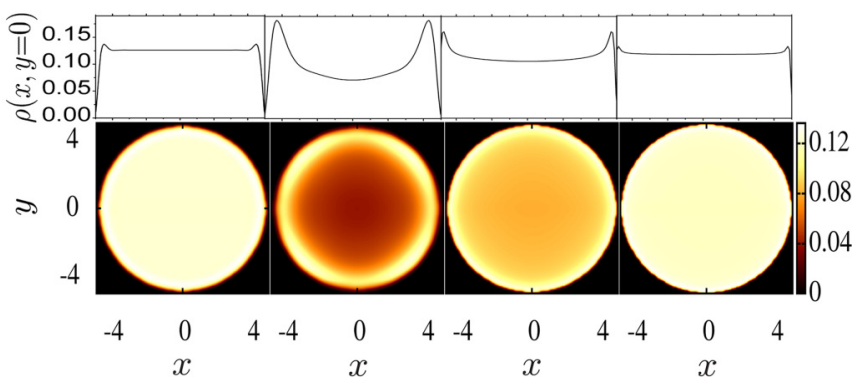

FIG. 3. (Color online) Two-dimensional dimensionless density distributions of the disk and the respective $1 \mathrm{D}$ cut at $y=0$, yielding $\rho(x, y=0)$, for (top) larger average density $\rho=7.95(a=2)$, for contact interactions, $n=1, n=2, n=3$, from left to right, and (bottom) smaller average density $\rho=1.27(a=5)$ at the same values of $n$. Darker and brighter colors indicate lower and higher density, respectively; cuts at $y=0$ are also shown. The $x$ and $y$ coordinates are dimensionless, scaled with the unit of length $l$.

the kinetic energy, leading to the small gradual decrease of $P_{\mathrm{TF}}$ with density. The difference from the mean-field behavior is, however, much more significant for $n=1$ and $n=2$, with the largest effect for $n=1$. Here, especially for $n=1$, the variation of $E_{\text {int }}$ with $\rho$ does not have a simple power-law form and overall increases slower than $E_{\text {kin }}$, leading to the rapid rise of $P_{\mathrm{TF}}$ at small densities. For example, when $n=1, E_{\text {int }} \sim \rho^{0.45}$ while $E_{\text {kin }} \sim \rho^{0.7}$. Similarly, for $n=2$, $E_{\text {int }} \sim \rho^{0.8}$ while $E_{\text {kin }} \sim \rho^{0.9}$.

\section{Density profiles}

The difference between the influence of the integrable and nonintegrable interactions on fragmentation can be connected to the spatial density profile $\rho(\mathbf{r}) \equiv \rho^{(1)}\left(\mathbf{r}^{\prime}=\mathbf{r} \mid \mathbf{r}\right)$. For large densities (small radius), the influence of the repulsive interactions for all values of $n$ is felt on the entire disk, leading to a higher density in the rim of the disk (see Fig. 3, top panel). As the radius increases (thus lowering $\rho$ ), the influence of the short-range interactions (contact, $n=3$ ) on the whole area of the disk is reduced because the radius becomes much larger than any length scale associated with the interactions, and the density is hence rendered homogeneous. The nonintegrable interactions ( $n=1,2)$, on the other hand, retain the long-range influence of the interaction for any radius of the disk. As a result, the equalization of densities does not occur, and the rim of the disk continues to have higher densities, which is clearly visible in the bottom panel of Fig. 3.

This difference manifests itself in the occupation of higher orbitals and thus the degree of fragmentation observed. The short range of integrable interactions leads to an essentially localized behavior, and long-range correlations between the bosons are very weak. Nonintegrable interactions, on the other hand, imply longer-range correlations: The sphere of influence of the interactions involves the contribution of a much larger number of bosons. A given boson thus interacts with all the bosons in the disk irrespective of the latter's radius and hence feels a stronger effective interaction. In more formal terms, the kernel of the matrix elements of the two-body interaction,

$$
W_{\alpha \beta \mu \nu}=\int d^{2} r d^{2} r^{\prime} \phi_{\alpha}(\boldsymbol{r}) \phi_{\beta}\left(\boldsymbol{r}^{\prime}\right) V_{\mathrm{int}}\left(\boldsymbol{r}-\boldsymbol{r}^{\prime}\right) \phi_{\mu}\left(\boldsymbol{r}^{\prime}\right) \phi_{\nu}(\boldsymbol{r}),
$$

has a larger support for smaller values of $n$ (longer ranges of interaction). Therefore, the integrals $W_{k s q l}$ are effectively larger, such that the system tends to minimize the energy by occupying several orbitals $\phi_{j}$, that is, through fragmentation.

\section{B. Comparison with the one-dimensional case}

While in $2 \mathrm{D}$, for contact interactions, we observe the absence of a change in fragmentation with density, in 1D there is a significant influence of the density, in line with what is known from exact diagonalization studies [34]. We compare a system of $N=100$ bosons in the disk geometry for the $2 \mathrm{D}$ case and $N=100$ bosons in a $1 \mathrm{D}$ "tube" with periodic boundary conditions.

\section{Long-range interactions}

Our simulations clearly reveal the difference between systems with long-range interactions in $1 \mathrm{D}$ and $2 \mathrm{D}$. In Fig. 4, we show the effect of the density $\rho^{1 \mathrm{D}}=\frac{N}{L}$ (where $L$ is the length of the 1D tube), on fragmentation $\mathcal{F}$ for a constant $V_{\text {eff }} \equiv \int V_{\text {int }}(R) d R=5.15$. Fragmentation decreases with density for all $n$. For $n=1$ in Eq. (4), the decrease with density is most rapid. For $n=2,3$ (which are integrable in

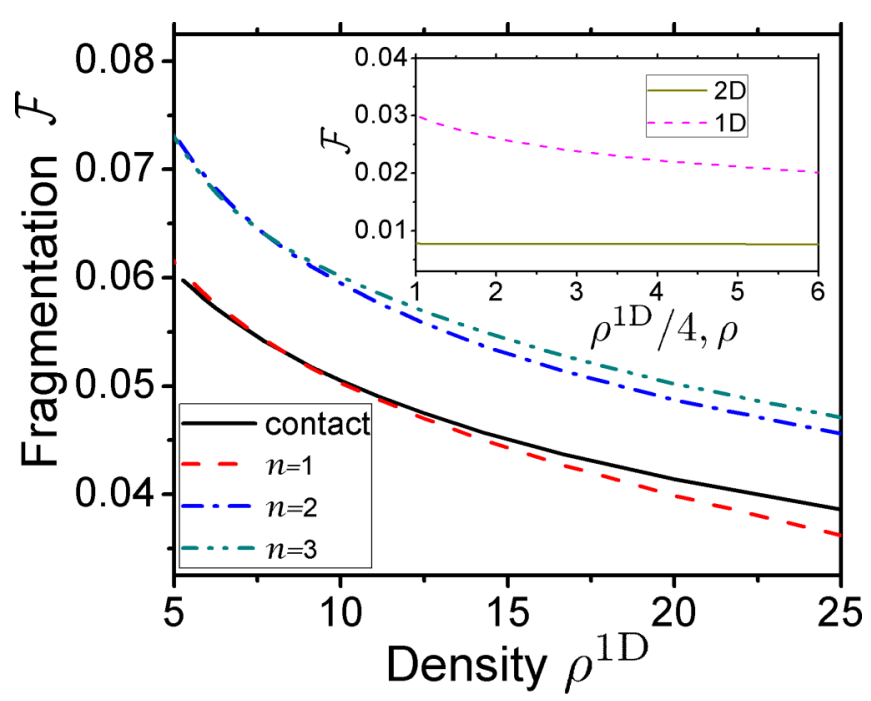

FIG. 4. (Color online) Variation of fragmentation with dimensionless density for $N=1001 \mathrm{D}$ bosons with contact interactions and long-range interactions. The inset shows the density dependence of fragmentation on dimensionality at equal density for $N=100$ $1 \mathrm{D}$ and $2 \mathrm{D}$ bosons, interacting with unit strength $g=1$. The $1 \mathrm{D}$ density $\rho^{1 \mathrm{D}}$ is scaled by $\frac{1}{4}$ to directly compare with the $2 \mathrm{D}$ range of densities $\rho$. 
1D), the decrease is similar, especially for larger densities. This is in complete accordance with our observations in the 2D bosonic gas: Concerning the dependence of $\mathcal{F}$ on $\rho$, integrable interactions demonstrate in either dimension similar behavior when compared to nonintegrable interactions.

\section{Contact interactions}

For contact interactions, in Fig. 4 (inset), we compare the variation of fragmentation with density for 1D and 2D gases for $N=100$ and $g=1$ (for the sake of a convenient comparison with the scale of the density of the $2 \mathrm{D}$ computations, we rescaled the $1 \mathrm{D}$ density $\rho^{1 \mathrm{D}}$ in the inset of Fig. 4 by a factor $\frac{1}{4}$ ). We observe a decrease of $\mathcal{F}$ for the 1D case with increasing density $\rho^{1 \mathrm{D}}$. The comparison to the $2 \mathrm{D}$ case with its absence of a significant variation of $\mathcal{F}$ with density again highlights the marginal nature of the two-dimensional system. In 3D, we anticipate that fragmentation is, on the one hand, very small $[48,49]$ and, on the other, decreases with decreasing density.

\section{NUMBER DEPENDENCE OF FRAGMENTATION}

Fragmentation is a mesoscopic phenomenon in the sense that for fixed interaction coupling, increasing $N$ while keeping all other parameters fixed always leads to a decrease in fragmentation. We have observed that this is a universal phenomenon and true for both integrable and nonintegrable interactions for the presently considered ground states of $1 \mathrm{D}$ and $2 \mathrm{D}$ bosonic gases contained in a single trap.

We illustrate in Fig. 5(a) how the fragmentation $\mathcal{F}$ varies in 2D with the number of particles $N$ for contact and long-range interactions $n=1,2,3$ [see Eqs. (3) and (4)] at a fixed $V_{\text {eff }}=2 \pi \times 11.6$; in $1 \mathrm{D}$ (not shown), a similar $N$-dependence of $\mathcal{F}$ is obtained. A decrease in fragmentation is observed for all cases. Although the mean-field interaction $V_{\mathrm{MF}}=(N-1) g$ increases with $N$ at constant coupling $g$, this does not lead to an increase in the fragmentation $\mathcal{F}$. Instead, we find that the particle number $N$ dominates over the effects of all other parameters affecting the fragmentation $\mathcal{F}$. That is, the variation of $\mathcal{F}$ with other parameters such as interaction strength $g$ and degree of long-rangedness parametrized by $n$ is subdominant in comparison. We stress that this qualitative functional number dependence is universal in that it is true regardless of densities, interaction strength, and other parameters. Note that this fact is not captured by a (single-parameter) variational theory [50], which demonstrates the sensitivity of fragmentation on the solutions of the manybody equations being fully self-consistent.

In order to assess the relative decrease of fragmentation $\mathcal{F}$, we plot in Fig. 5(b) the $N$ dependence of $\mathcal{F}$ relative to the reference value set at $N=N_{0}=10$. The contact interaction curve shows the smallest decrease with $N$, closely followed by $n=1$. The cases with $n=2$ and $n=3$ show very similar dependence and decay more rapidly with $N$ compared to $n=1$. For constant interaction coupling, the decrease of the fragmentation degree is well approximated by $\mathcal{F}(N) / \mathcal{F}\left(N_{0}\right) \propto$ $N^{-1 / 2}$ [see Fig. 5(b)]. It is noteworthy that this $N^{-\frac{1}{2}}$ scaling of fragmentation degree holds for all power laws of interaction we consider. We finally mention that, when the effective mean-

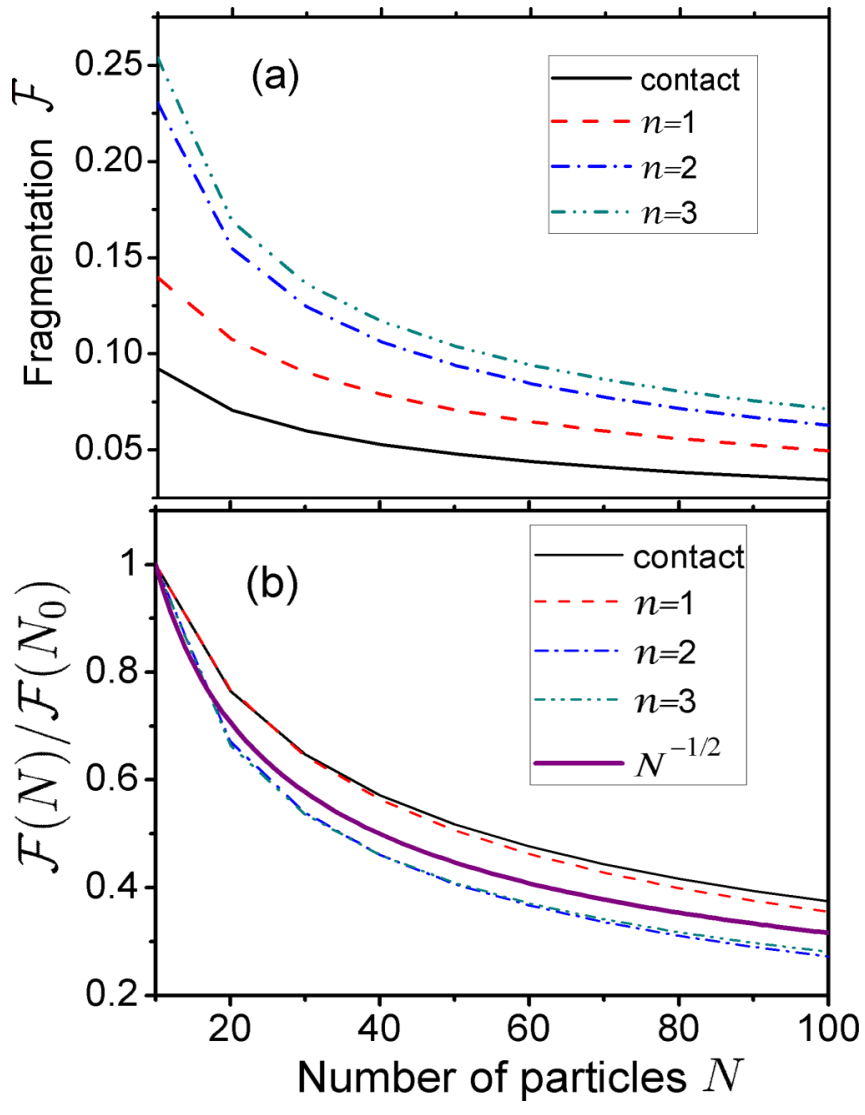

FIG. 5. (Color online) (a) Number dependence of fragmentation for contact and long-range interactions for 2D. (b) Number dependence with fragmentation normalized to its value at $N_{0}=10$.

field interactions $V_{\mathrm{MF}}=(N-1) g$ are kept constant, then $\mathcal{F}$ falls off much faster (not shown), approximately as $1 / N$.

\section{CONCLUSION}

Using condensate fragmentation as a diagnostic tool, we have assessed the degree to which an interacting system of bosons can be considered to be dilute and therefore describable by mean-field theory and a single macroscopically occupied orbital. The diluteness parameter in a $2 \mathrm{D}$ gas is essentially independent of density [8]. We have confirmed that this behavior is reflected by an approximate independence on density of the degree of fragmentation $\mathcal{F}$ for integrable interactions. However, for nonintegrable interactions, a significant dependence of $\mathcal{F}$ on the density, namely, a significant decrease in the degree of fragmentation with increasing density, is obtained, and this dependence increases for longer-range interactions. Due to the fact that fragmentation in a single-well trap is a genuine many-body phenomenon, the degree of fragmentation thus represents a quantitative measure of to what extent long-range interactions lead to strong correlations in two spatial dimensions. We have also found that in the large $N$ limit, in a self-consistent approach, mean-field theory again becomes valid: The degree of fragmentation, to a good approximation, universally decreases as $N^{-\frac{1}{2}}$ when only $N$ is varied. Fragmentation in single traps is therefore a mesoscopic many-body phenomenon. 
We finally stress again that the detection of the fragmentation of interacting Bose gases into two incoherent macroscopic pieces in single-well traps yields more information about the many-body physics than its double-well counterpart, where the emergence of many-body correlations is largely predetermined by spatially splitting the system and hence by the one-body part of the Hamiltonian. A concrete analysis of single-well fragmentation can, for example, be performed by measuring

[1] C. J. Pethick and H. Smith, Bose-Einstein Condensation in Dilute Gases (Cambridge University Press, Cambridge, 2002).

[2] N. N. Bogoliubov, Selected Works II: Quantum and Statistical Mechanics (Gordon and Breach, New York, 1991).

[3] L. P. Pitaevskiǔ and S. Stringari, Bose-Einstein Condensation (Clarendon, Oxford, 2003).

[4] E. Wigner, On the interaction of electrons in metals, Phys. Rev. 46, 1002 (1934).

[5] L. L. Foldy, Charged Boson Gas, Phys. Rev. 124, 649 (1961).

[6] G. E. Astrakharchik and M. D. Girardeau, Exact ground-state properties of a one-dimensional Coulomb gas, Phys. Rev. B 83, 153303 (2011).

[7] V. Dunjko, V. Lorent, and M. Olshanii, Bosons in cigar-shaped traps: Thomas-Fermi regime, Tonks-Girardeau regime, and in between, Phys. Rev. Lett. 86, 5413 (2001).

[8] D. S. Fisher and P. C. Hohenberg, Dilute Bose gas in two dimensions, Phys. Rev. B 37, 4936 (1988).

[9] N. D. Mermin and H. Wagner, Absence of ferromagnetism or antiferromagnetism in one- or two-dimensional isotropic Heisenberg models, Phys. Rev. Lett. 17, 1133 (1966).

[10] P. C. Hohenberg, Existence of long-range order in one and two dimensions, Phys. Rev. 158, 383 (1967).

[11] U. R. Fischer, Existence of long-rang order for trapped Interacting Bosons, Phys. Rev. Lett. 89, 280402 (2002).

[12] Y. Castin, Simple theoretical tools for low dimension Bose gases, J. Phys. IV France 116, 89 (2004).

[13] M. Schick, Two-dimensional system of hard-core Bosons, Phys. Rev. A 3, 1067 (1971).

[14] A. Posazhennikova, Weakly interacting, dilute Bose gases in 2D, Rev. Mod. Phys. 78, 1111 (2006).

[15] R. B. Laughlin, Anomalous quantum hall effect: An incompressible quantum fluid with fractionally charged excitations, Phys. Rev. Lett. 50, 1395 (1983).

[16] H. L. Störmer, D. C. Tsui, and A. C. Gossard, The fractional quantum Hall effect, Rev. Mod. Phys. 71, S298 (1999).

[17] M. A. Baranov, Theoretical progress in many-body physics with ultracold dipolar gases, Phys. Rep. 464, 71 (2008).

[18] U. R. Fischer, Stability of quasi-two-dimensional Bose-Einstein condensates with dominant dipole-dipole interactions, Phys. Rev. A 73, 031602(R) (2006).

[19] A. U. J. Lode and M. C. Tsatsos, The recursive time-dependent Hartree for bosons package, http://ultracold.org.

[20] O. Penrose and L. Onsager, Bose-Einstein condensation and liquid Helium, Phys. Rev. 104, 576 (1956).

[21] A. J. Leggett, Bose-Einstein condensation in the alkali gases: Some fundamental concepts, Rev. Mod. Phys. 73, 307 (2001). density-density correlations after time-of-flight expansion, as detailed in [33].

\section{ACKNOWLEDGMENTS}

The research of U.R.F. and B.C. was supported by the NRF of Korea, Grant No. 2014R1A2A2A01006535, and the BK21 Program. A.U.J.L. acknowledges financial support by the Swiss SNF and the NCCR Quantum Science and Technology.
[22] E. J. Mueller, T.-L. Ho, M. Ueda, and G. Baym, Fragmentation of Bose-Einstein condensates, Phys. Rev. A 74, 033612 (2006).

[23] O. E. Alon, A. I. Streltsov, and L. S. Cederbaum, Multiconfigurational time-dependent Hartree method for bosons: Many-body dynamics of bosonic systems, Phys. Rev. A 77, 033613 (2008).

[24] P. Bader and U. R. Fischer, Fragmented many-body ground states for scalar Bosons in a single trap, Phys. Rev. Lett. 103, 060402 (2009).

[25] O. E. Alon and L. S. Cederbaum, Pathway from condensation via fragmentation to fermionization of cold Bosonic systems, Phys. Rev. Lett. 95, 140402 (2005).

[26] A. I. Streltsov, Quantum systems of ultracold bosons with customized interparticle interactions, Phys. Rev. A 88, 041602(R) (2013).

[27] S. Klaiman, A. U. J. Lode, A. I. Streltsov, L. S. Cederbaum, and O. E. Alon, Breaking the resilience of a two-dimensional Bose-Einstein condensate to fragmentation, Phys. Rev. A 90, 043620 (2014).

[28] M. R. Andrews, C. G. Townsend, H.-J. Miesner, D. S. Durfee, D. M. Kurn, W. Ketterle, Observation of interference between two Bose condensates, Science 275, 637 (1997).

[29] R. W. Spekkens and J. E. Sipe, Spatial fragmentation of a BoseEinstein condensate in a double-well potential, Phys. Rev. A 59, 3868 (1999).

[30] C. Orzel, A. K. Tuchman, M. L. Fenselau, M. Yasuda, M. A. Kasevich, Squeezed states in a Bose-Einstein condensate, Science 291, 2386 (2001).

[31] M. Greiner, O. Mandel, T. Esslinger, T. W. Hänsch, and I. Bloch, Quantum phase transition from a superfluid to a Mott insulator in a gas of ultracold atoms, Nature (London) 415, 39 (2002).

[32] T. W. Hänsch, I. Bloch, and T. Esslinger, Probing first-order spatial coherence of a Bose-Einstein condensate, J. Mod. Opt. 47, 2725 (2000).

[33] M.-K. Kang and U. R. Fischer, Revealing single-trap condensate fragmentation by measuring density-density correlations after time of flight, Phys. Rev. Lett. 113, 140404 (2014).

[34] M. A. Cazalilla, R. Citro, T. Giamarchi, E. Orignac, and M. Rigol, One dimensional bosons: From condensed matter systems to ultracold gases, Rev. Mod. Phys. 83, 1405 (2011).

[35] B. Paredes, P. Fedichev, J. I. Cirac, and P. Zoller, $\frac{1}{2}$-Anyons in small atomic Bose-Einstein condensates, Phys. Rev. Lett. 87, 010402 (2001).

[36] A. Khare and K. Ray, A quantum many-body problem in two dimensions: Ground state, Phys. Lett. A 230, 139 (1997).

[37] J. Dukelsky and P. Schuck, Condensate fragmentation in a new exactly solvable model for confined Bosons, Phys. Rev. Lett. 86, 4207 (2001). 
[38] L. Cohen and C. Lee, Exact reduced density matrices for a model problem, J. Math. Phys. 26, 3105 (1985).

[39] J. Yan, Harmonic interaction model and its application in BoseEinstein condensation, J. Stat. Phys. 113, 623 (2003).

[40] A. U. J. Lode, K. Sakmann, O. E. Alon, L. S. Cederbaum, and A. I. Streltsov, Numerically exact quantum dynamics of bosons with time-dependent interactions of harmonic type, Phys. Rev. A 86, 063606 (2012).

[41] A. U. J. Lode, Tunneling Dynamics in Open Ultracold Bosonic Systems, Springer Theses (Springer, Heidelberg, 2015).

[42] A. L. Gaunt, T. F. Schmidutz, I. Gotlibovych, R. P. Smith, and Z. Hadzibabic, Bose-Einstein condensation of atoms in a uniform potential, Phys. Rev. Lett. 110, 200406 (2013).

[43] L. Chomaz, L. Corman, T. Bienaimé, R. Desbuquois, C. Weitenberg, S. Nascimbène, J. Beugnon, and J. Dalibard, Emergence of coherence via transverse condensation in a uniform quasi-two-dimensional Bose gas, Nat. Commun. 6, 6162 (2015).

[44] R. A. Doganov, S. Klaiman, O. E. Alon, A. I. Streltsov, and L. S. Cederbaum, Two trapped particles interacting by a finite-range two-body potential in two spatial dimensions, Phys. Rev. A 87, 033631 (2013).

[45] C. N. Friedman, Perturbations of the Schrödinger equation by potentials with small support, J. Funct. Anal. 10, 346 (1972).

[46] Geometry of the Time-Dependent Variational Principle in Quantum Mechanics, edited by P. Kramer and M. Saraceno, Lecture Notes in Physics Vol. 140 (Springer, Heidelberg, 1981).

[47] Y. Yu, Z. Luo, and Z. Wang, Effects of dipole-dipole interaction between cigar-shaped BECs of cold alkali atoms: Towards inverse-squared interactions, J. Phys. Condens. Matter 26, 305402 (2014).

[48] P. Bader and U. R. Fischer, Stability of spherically trapped threedimensional Bose-Einstein condensates against macroscopic fragmentation, Phys. Rev. A 87, 023632 (2013).

[49] T. Wells, A. U. J. Lode, V. S. Bagnato, and M. C. Tsatsos, Vortex reconnections in anisotropic trapped three-dimensional Bose-Einstein condensates, J. Low Temp. Phys. 180, 133 (2015).

[50] U. R. Fischer and P. Bader, Interacting trapped bosons yield fragmented condensate states in low dimensions, Phys. Rev. A 82, 013607 (2010). 